\title{
Aortic shear stress in patients with bicuspid aortic valve with stenosis and insufficiency
}

\author{
Yan Shan, MD, PhD, ${ }^{\mathrm{a}}$ Jun Li, MD, PhD, ${ }^{\mathrm{b}}$ Yongshi Wang, MD, ${ }^{\mathrm{a}, \mathrm{b}}$ Boting Wu, MD, PhD, \\ Alex J. Barker, PhD, ${ }^{\mathrm{c}}$ Michael Markl, PhD, ${ }^{\mathrm{c}, \mathrm{d}}$ Chunsheng Wang, MD, ${ }^{\mathrm{b}}$ Xiaolin Wang, MD, ${ }^{\mathrm{a}}$ and \\ Xianhong Shu, MD, $\mathrm{PhD}^{\mathrm{a}, \mathrm{b}}$
}

\begin{abstract}
Objectives: Bicuspid aortic valve, characterized by valve malformation and risk for aortopathy, displays profound alteration in systolic aortic outflow and wall shear stress distribution. The present study performed 4-dimensional flow magnetic resonance imaging in patients with bicuspid aortic valve with right-left cusp fusion, focusing on the impact of valve function on hemodynamic status within the ascending aorta.
\end{abstract}

Methods: Four-dimensional flow magnetic resonance imaging was performed in 50 subjects with right-left bicuspid aortic valve and 15 age- and aortic sizematched controls with tricuspid aortic valve. Patients with bicuspid aortic valve were categorized into 3 groups according to their aortic valve function as follows: bicuspid aortic valve with no more than mild aortic valve dysfunction (bicuspid aortic valve control, $\mathrm{n}=20$ ), bicuspid aortic valve with severe aortic insufficiency $(\mathrm{n}=15)$, and bicuspid aortic valve with severe aortic stenosis $(\mathrm{n}=15)$.

Results: All patients with right-left bicuspid aortic valve exhibited peak wall shear stress at the right-anterior position of the ascending aorta (bicuspid aortic valve vs trileaflet aortic valve at the right-anterior position: $0.91 \pm 0.23 \mathrm{~N} / \mathrm{m}^{2}$ vs $0.43 \pm 0.12 \mathrm{~N} / \mathrm{m}^{2}, P<.001$ ) with no distinct alteration between bicuspid aortic valve with severe aortic insufficiency and bicuspid aortic valve with severe aortic stenosis. The predominance of dilatation involving the tubular ascending aorta $(82 \%$, type 2 aortopathy) persisted, with or without valve dysfunction. Compared with bicuspid aortic valve control subjects, the bicuspid aortic valve with severe aortic insufficiency group displayed universally elevated wall shear stress $\left(0.75 \pm 0.12 \mathrm{~N} / \mathrm{m}^{2}\right.$ vs $\left.0.57 \pm 0.09 \mathrm{~N} / \mathrm{m}^{2}, P<.01\right)$ in the ascending aorta, which was associated with elevated cardiac stroke volume $(P<.05)$. The bicuspid aortic valve with severe aortic stenosis group showed elevated flow eccentricity in the form of significantly increased standard deviation of circumferential wall shear stress, which correlated with markedly increased peak aortic valve velocity $(P<.01)$.

Conclusions: The location of peak aortic wall shear stress and type of aortopathy remained homogeneous among patients with right-left bicuspid aortic valve irrespective of valve dysfunction. Severe aortic insufficiency or stenosis resulted in further elevated aortic wall shear stress and exaggerated flow eccentricity. ( $\mathrm{J}$ Thorac Cardiovasc Surg 2017;153:1263-72)

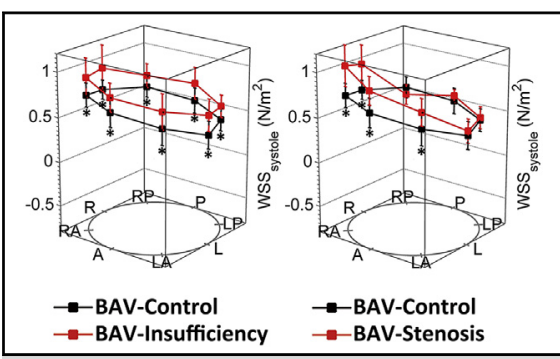

Wall shear stress distribution features in BAV insufficiency and stenosis.

Central Message

Hemodynamic alterations revealed by 4D flow MRI in subjects with BAV indicated additional aortic WSS elevation and flow eccentricity exaggeration due to severe aortic insufficiency or aortic stenosis.

\section{Perspective}

Patients with BAV with aortic insufficiency tended to have a higher risk for progressive ascending aorta dilatation and adverse aortic events after isolated aortic valve replacement surgery compared with their aortic stenosis counterparts. Spatiotemporal characteristics of distorted flow in the setting of aortic valve dysfunction, which could have long-term consequences in affected aortic wall tissue, and hemodynamic markers derived from MRI metrics represent promising developments toward establishing noninvasive indicators for risk stratification and management of patients with BAV.

See Editorial Commentary page 1273.

See Editorial page 1261.

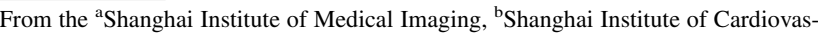
cular Diseases, 'Department of Transfusion, Zhongshan Hospital Fudan University, Shanghai, China; ' Department of Radiology, Feinberg School of Medicine, Northwestern University, Chicago; and ${ }^{\mathrm{d}}$ Department of Biomedical Engineering, McCormick School of Engineering, Northwestern University, Evanston, Ill.

The study was funded by National Natural Science Foundation of China Grant No. 81300232 (to Y.W.); Shanghai Municipal Committee of Science and Technology Grant No. 16ZR1433100 (to X.W.); Shanghai Municipal Health Bureau Grant No. 20154 Y0030 (to Y.W.); National Institutes of Health National Heart, Lung, and Blood Institute Grants R01HL115828 (to M.M.) and K25HL119608 (to A.J.B.); and Zhongshan Hospital Grant No. 2015ZSYXQN02 (to Y.S.).
}

Y.S. and J.L. contributed equally to this manuscript.

Received for publication Sept 7, 2016; revisions received Dec 13, 2016; accepted for publication Dec 27, 2016; available ahead of print March 6, 2017

Address for reprints: Yongshi Wang, MD, Shanghai Institute of Cardiovascular Diseases, Zhongshan Hospital Fudan University, 180 Fenglin Rd, Shanghai 200032, China (E-mail: wang.yongshi@zs-hospital.sh.cn).

$0022-5223 / \$ 36.00$

Copyright (C) 2017 by The American Association for Thoracic Surgery

http://dx.doi.org/10.1016/j.jtcvs.2016.12.059 


\begin{tabular}{|c|c|}
\hline Abbreviations and & Acronyms \\
\hline AI & $=$ aortic insufficiency \\
\hline AS & $=$ aortic stenosis \\
\hline BAV & $=$ bicuspid aortic valve \\
\hline CMR & $=$ cardiac magnetic resonance \\
\hline CTL & $=$ control \\
\hline $4 \mathrm{D}$ & $=4$-dimensional \\
\hline LP & $=$ left posterior \\
\hline MRI & $=$ magnetic resonance imaging \\
\hline RA & $=$ right anterior \\
\hline ratio $\mathrm{WSS}_{\max -\mathrm{mir}}$ & $\begin{aligned} & \mathrm{n}= \text { ratio of WSS between the } \\
& \text { maximum and minimum } \\
& \text { position }\end{aligned}$ \\
\hline R-L & $=$ right left \\
\hline TAV & $=$ tricuspid aortic valve \\
\hline $3 \mathrm{D}$ & $=3$-dimensional \\
\hline $2 \mathrm{D}$ & $=2$-dimensional \\
\hline WSS & $=$ wall shear stress \\
\hline WSS-SD $8 \mathrm{P}$ & $\begin{aligned}= & \text { standard deviation of } \\
& \text { circumferential WSS across } 8 \\
& \text { anatomic positions }\end{aligned}$ \\
\hline
\end{tabular}

Scanning this QR code will take you to a supplemental video for the article.

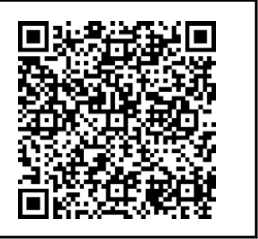

Bicuspid aortic valve (BAV), the most common congenital cardiac disorder in adults, is associated with a heterogeneous expression of aortic diseases, such as ascending aortic dilatation, aneurysm, and dissection. ${ }^{1-4}$ Both genetic and hemodynamic origins of these complications have been proposed, and there exists a "fate versus flow" controversy regarding the development of BAV aortopathy. ${ }^{5}$ As a result, the inherent heterogeneity of BAV aortopathy currently is not fully integrated into the existing knowledge infrastructure and patient management. The constantly changing practice guidelines for BAV aortopathy also need to be revised and redirected into the realm of individualized decision making. ${ }^{6,7}$ Therefore, collective efforts, for example, as proposed by the International Bicuspid Aortic Valve Consortium, ${ }^{8}$ should be made to address the variability in genetic basis, hemodynamics, and aortic wall extracellular matrix abnormalities to meet the contemporary focus on precision medicine.

Time-resolved 4-dimensional magnetic resonance imaging (4D flow MRI) offers a novel approach to assess hemodynamic alterations in BAV. ${ }^{9}$ Previous studies have shown that BAV and its cusp fusion type can lead to distinct changes in systolic aortic outflow and wall shear stress (WSS) distribution patterns, resulting in different regional histology, proteolytic activity, and unfavorable ascending aortic remodeling. ${ }^{10-13}$ It has been demonstrated in recent cohort studies that patients with BAV with aortic insufficiency tended to have a higher risk for progressive ascending aorta dilatation and adverse aortic events after isolated aortic valve replacement surgery compared with their aortic stenosis counterparts. ${ }^{14-16}$ However, data are limited on systolic aortic outflow and WSS distribution as a function of aortic valve dysfunction, such as stenosis or insufficiency.

The present study performed 4D flow MRI in patients with right-left (R-L) cusp fusion type BAV with severe aortic stenosis (AS) or insufficiency (AI), and compared 3-dimensional (3D) aortic outflow pattern, WSS, and WSS eccentricity with BAV and TAV control subjects with similar aortic sizes. Our aim was to investigate the hypothesis that aortic valve dysfunction exerts additional but distinct alterations on systolic aortic outflow and WSS distribution patterns in the BAV ascending aorta, which might be attributed to characteristic physiologic changes of AS and AI, and could facilitate individualized surgical strategies for BAV aortopathy.

\section{MATERIALS AND METHODS \\ Study Population}

Patients with BAV with an R-L cusp fusion type were screened from the cardiology clinic of our institution by transthoracic echocardiography and recruited for 4D flow MRI from December 2014 to February 2016. Subjects with BAV were categorized into 3 groups according to their aortic valve function as follows: patients with BAV with no more than mild aortic stenosis or insufficiency (BAV-CTL group, $n=20$ ); patients with BAV with severe aortic insufficiency but no more than mild aortic stenosis (BAV-AI group, $\mathrm{n}=15$ ); and patients with BAV with severe aortic stenosis but no more than mild aortic insufficiency (BAV-AS group, $\mathrm{n}=15$ ). Ageand aortic size-matched volunteers with tricuspid aortic valve and no more than mild aortic stenosis or insufficiency were recruited as controls (TAV-CTL group, $\mathrm{n}=15$ ). According to American College of Cardiology/ American Heart Association practice guidelines, ${ }^{17}$ severe aortic insufficiency was defined as jet width/left ventricular outflow tract greater than $65 \%$ or vena contracta greater than $0.6 \mathrm{~cm}$ or regurgitant fraction greater than $50 \%$; severe aortic stenosis was defined as mean transaortic pressure gradient greater than $40 \mathrm{~mm} \mathrm{Hg}$ or aortic valve area less than $1.0 \mathrm{~cm}^{2}$. Exclusion criteria consisted of previous cardiac surgery, infective endocarditis, acute aortic dissection, or other severe congenital cardiac defects (ie, coarctation of the aorta, tetralogy of Fallot, ventricular septal defect, and supra-aortic stenosis). The present study conformed to the principles outlined in the Declaration of Helsinki and was approved by the ethics committee of Zhongshan Hospital Fudan University, Shanghai, China.

\section{Echocardiography}

Transthoracic echocardiographic images were acquired in all subjects with a commercially available system (iE33, Philips Medical Systems, Bothell, Wash). Two-dimensional and Doppler echocardiography were performed at rest in the left lateral decubitus position according to a uniform and standardized protocol based on the American College of Cardiology/American Heart Association practice guidelines and American 
Society of Echocardiography recommendations. ${ }^{17,18}$ All image data were analyzed by an experienced echocardiologist blinded to the results of the MRI study. The diagnosis of BAV and cusp fusion type was confirmed in accordance to the classification by Sievers and Schmidtke ${ }^{19}$ by transthoracic or transesophageal echocardiography in a short-axis view of the aortic valve with only 2 valve cusps clearly identified. Peak aortic valve velocity and mean transvalvular gradient were assessed by continuous-wave Doppler echocardiography in the apical long-axis and 5-chamber view, and aortic valve area was calculated via the continuity equation. Aortic regurgitation was graded according to the standard methods by color Doppler in the parasternal long-axis and short-axis view, as well as in the apical long-axis and 5-chamber view.

\section{Magnetic Resonance Imaging}

All subjects underwent cardiac MRI on a commercially available 3.0 Tesla system (Verio, Siemens Medical Systems, Erlangen, Germany). Aortic diameters at the level of aortic sinus, sinotubular junction, midascending, and proximal descending aorta at the level of the pulmonary artery were obtained from axial half-Fourier single-shot turbo spin-echo scan sequence imaging of the thorax. Dilatation of aortic segments (root, ascending aorta, and transverse arch) was defined as diameter greater than $40 \mathrm{~mm} .{ }^{20}$ In addition, the phenotype of BAV aortopathy was categorized as follows: type 0 , normal aorta; type 1 , dilated only at the aortic root; type 2, aortic enlargement involving the tubular portion of ascending aorta; type 3, diffuse involvement of both the entire ascending aorta and the transverse aortic arch. ${ }^{11}$ The assessment of aortic blood flow was facilitated by 4D flow MRI with 3-directional velocity encoding. Data were acquired during free breathing using respiratory and prospective electrocardiogram gating in a sagittal oblique 3D volume covering the entire thoracic aorta. Pulse sequence parameters were as follows: flip angle $=7^{\circ}$, spatial resolution $=(2.2-2.6) \times(1.8-2.1) \times(2.2-2.5) \mathrm{mm}^{3}$, temporal resolution $=40.8 \mathrm{~ms}$. Velocity encoding was adjusted to minimize velocity aliasing ranging from 150 to $400 \mathrm{~cm} / \mathrm{s}$ based on 2-dimensional (2D) phasecontrast MRI scout measurements. 4D flow MRI acquisition time varied from 12 to 20 minutes depending on navigator efficiency and individual heart rate.

\section{Four-Dimensional Flow Data Analysis}

4D flow MRI preprocessing included noise filtering, correction for eddy currents, and velocity aliasing with custom-built software programmed in MatLab (The MathWorks Inc, Natick, Mass). 3D PC magnetic resonance angiography was derived from $4 \mathrm{D}$ flow MRI data and used to visualize 3D aortic flow patterns by velocity-coded vectors and streamlines (EnSight, CEI, Apex, NC) at peak flow systole. Measurement planes were manually placed perpendicular to the long axis of the aorta at defined anatomic landmarks in the proximal ascending aorta directly distal to the sinotubular junction, mid-ascending aorta, distal ascending aorta at the origin of the brachiocephalic trunk, and proximal descending aorta (Figure 1) based on previously published criteria. ${ }^{11}$ Aortic lumen contours were manually delineated at all time frames with the use of customized MatLab software to calculate peak velocity and time-resolved WSS distribution (Appendix E1). Systolic WSS was calculated in 8 anatomic positions (anterior, left anterior, left, left posterior [LP], posterior, right posterior, right, and right anterior [RA]) and averaged over the proceeding and 4 subsequent time steps after the peak systolic flow. Standard deviation of circumferential WSS across 8 anatomic positions (WSS-SD $8 \mathrm{P}$ ) and ratio of WSS between the maximum and minimum position (ratio WSS $_{\text {max-min }}$ ) at each measurement plane were calculated as previously described. ${ }^{13}$ As confirmed from the maximum RA-LP WSS values in the healthy control populations, a WSS eccentricity threshold defined as RA-LP greater than $0.2 \mathrm{~N} / \mathrm{m}^{2}$ was used to categorize the flow as eccentric. ${ }^{10}$ Circumferentially averaged WSS was calculated as the mean over all 8 positions. WSS measurements were repeated in 20 subjects ( 10 volunteers with TAV, 10 patients with BAV) 1 week later to assess the reproducibility. The intraclass

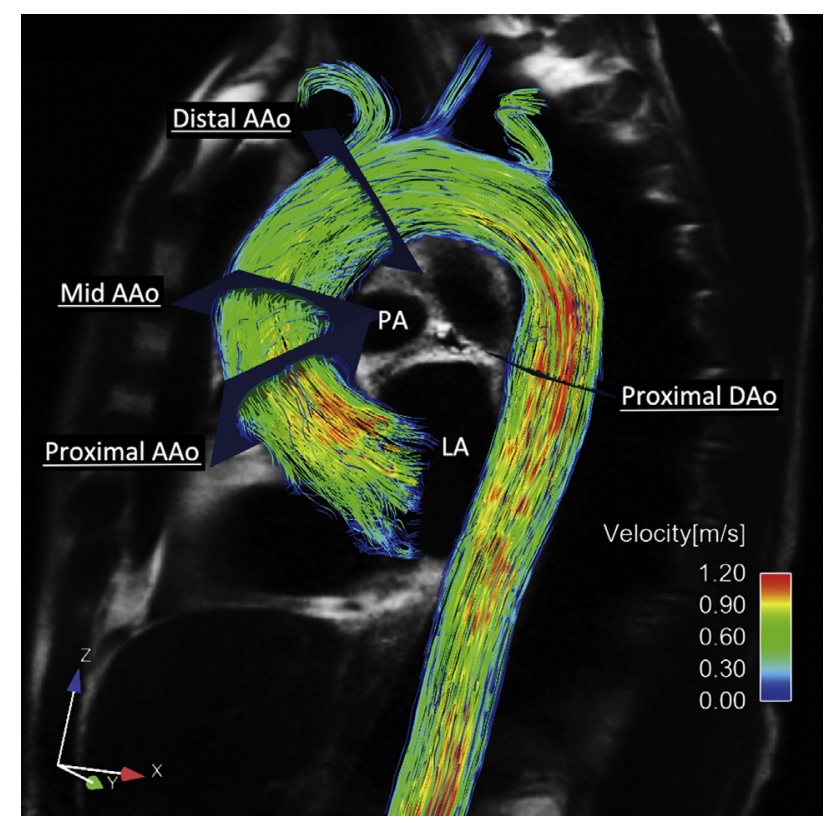

FIGURE 1. Representative images and analytic planes for 4D flow MRI. $A A o$, Ascending aorta; DAo, descending aorta.

correlation coefficients between 2 measurements for systolic WSS, WSS-SD $_{8 \mathrm{~B}}$ and ratio $\mathrm{WSS}_{\text {max-min }}$ were $0.73,0.78$, and 0.63 , respectively.

\section{Statistical Analysis}

Analysis was performed with SPSS 12.0 software (SPSS Inc, Chicago, Ill). Data were reported as mean \pm standard deviation or medians (interquartile ranges) for continuous variables and as frequencies (percentages) for categoric variables. The Shapiro-Wilk test was used to evaluate the normal distribution of continuous parameters. Differences between the 2 groups were assessed by the Student $t$ test (Gaussian distribution) or Mann-Whitney $U$ test (non-Gaussian distribution). Multiple comparisons were analyzed using 1-way analysis of variance (Gaussian distribution) or Kruskal-Wallis $\mathrm{H}$ analysis (non-Gaussian distribution). Differences in percentages were evaluated using the chi-square tests or Fisher exact tests. Correlation was assessed by Pearson's correlation coefficient. The level of significance for pairwise comparisons was adjusted when multiple comparisons were performed $(P=.05 / 4=.0125)$. Statistical significance was defined as 2-sided $P<.05$.

\section{RESULTS}

\section{Baseline Characteristics}

Patients with BAV demonstrated similar aortic diameter, as well as distribution of gender, comorbid condition, and medication status. Compared with the BAV-CTL group, the BAV-AI group had elevated stroke volume $(P<.001)$, higher systolic blood pressure $(P=.002)$, greater transvalvular pressure gradient $(P=.002)$, and increased aortic sinus diameter $(P=.020)$. The BAV-AS group demonstrated a marked reduction in aortic valve area $(P<.001)$, higher peak aortic valve velocity $(P<.001)$, greater transvalvular pressure gradient $(P<.001)$, and older age $(P=.005)$ (Table 1$)$.

Type 2 aortopathy, defined as aortic enlargement involving the tubular portion of ascending aorta, was the 
TABLE 1. Baseline characteristics of right-left bicuspid aortic valve cases and tricuspid aortic valve volunteers

\begin{tabular}{|c|c|c|c|c|}
\hline & \multicolumn{2}{|c|}{ Control group } & \multicolumn{2}{|c|}{ Study group } \\
\hline & TAV-CTL & BAV-CTL & BAV-AI & BAV-AS \\
\hline $\mathrm{n}$ (female) & $15(6)$ & $20(6)$ & $15(2)$ & $15(7)$ \\
\hline Age, y & $51 \pm 9$ & $47 \pm 11$ & $46 \pm 12$ & $57 \pm 7^{*}$ \\
\hline Body surface area, $\mathrm{m}^{2}$ & $1.78 \pm 0.12$ & $1.79 \pm 0.11$ & $1.79 \pm 0.13$ & $1.76 \pm 0.12$ \\
\hline \multicolumn{5}{|l|}{ Comorbid conditions, n (\%) } \\
\hline Hypertension & $3(20.0)$ & $3(15.0)$ & $3(20.0)$ & $2(13.3)$ \\
\hline Diabetes & $1(6.7)$ & 0 & 0 & $1(6.7)$ \\
\hline History of coronary artery disease & 0 & 0 & 0 & $1(6.7)$ \\
\hline Systolic blood pressure, $\mathrm{mm} \mathrm{Hg}$ & $124 \pm 10$ & $122 \pm 11$ & $138 \pm 15^{*}$ & $126 \pm 10$ \\
\hline Diastolic blood pressure, $\mathrm{mm} \mathrm{Hg}$ & $76 \pm 9$ & $75 \pm 9$ & $71 \pm 13$ & $76 \pm 9$ \\
\hline Beta-blocker therapy, n (\%) & 0 & $3(15.0)$ & $2(13.3)$ & $2(13.3)$ \\
\hline ACEI/ARB therapy, n (\%) & 0 & 0 & $2(13.3)$ & $2(13.3)$ \\
\hline LVEF (\%) & $63.1 \pm 3.7$ & $63.9 \pm 4.9$ & $60.7 \pm 6.3$ & $62.0 \pm 4.7$ \\
\hline \multicolumn{5}{|l|}{ Aortic valve features } \\
\hline Aortic valve area, $\mathrm{cm}^{2}$ & $2.5(2.4-2.7)$ & $2.4(2.2-2.6)$ & $2.6(2.4-2.8)$ & $0.8(0.7-0.9)^{*}$ \\
\hline Peak velocity, $\mathrm{m} / \mathrm{s}$ & $1.4 \pm 0.1$ & $1.7 \pm 0.4$ & $2.1 \pm 0.4$ & $4.6 \pm 0.7 *$ \\
\hline Mean gradient, $\mathrm{mm} \mathrm{Hg}$ & $5.4(3.8-6.2)$ & $6.3(5.0-8.0)$ & $10.2(7.2-12.0)^{*}$ & $46.1(41.8-57.3) *$ \\
\hline Stroke volume, $\mathrm{mL}$ & $54.3 \pm 7.5$ & $55.3 \pm 7.9$ & $107.3 \pm 13.8^{*}$ & $59.1 \pm 5.7$ \\
\hline \multicolumn{5}{|l|}{ Aortic diameters, mm } \\
\hline Aortic sinus & $33.9 \pm 3.3$ & $34.6 \pm 3.2$ & $38.0 \pm 4.3$ & $34.1 \pm 3.1$ \\
\hline Sinotubular junction & $29.8 \pm 3.7$ & $30.9 \pm 3.5$ & $31.8 \pm 4.0$ & $30.2 \pm 3.3$ \\
\hline Mid-ascending aorta & $38.8 \pm 4.8$ & $39.8 \pm 5.1$ & $40.4 \pm 5.8$ & $41.0 \pm 5.3$ \\
\hline Proximal descending aorta & $23.4 \pm 3.2$ & $24.4 \pm 3.1$ & $23.3 \pm 2.4$ & $22.5 \pm 2.6$ \\
\hline \multicolumn{5}{|l|}{ Aortopathy, n (\%) } \\
\hline Type 0 & $8(53.3)$ & $10(50.0)$ & $6(40.0)$ & $7(46.7)$ \\
\hline Type 1 & $1(6.7)$ & $1(5.0)$ & $1(6.7)$ & 0 \\
\hline Type 2 & $5(33.3)$ & $8(40.0)$ & $7(46.7)$ & $7(46.7)$ \\
\hline Type 3 & $1(6.7)$ & $1(5.0)$ & $1(6.7)$ & $1(6.7)$ \\
\hline
\end{tabular}

All continuous data are presented as mean \pm standard deviation or as median (interquartile range). $T A V$, Tricuspid aortic valve; $C T L$, control; $B A V$, bicuspid aortic valve; $A I$, aortic insufficiency; $A S$, aortic stenosis. *Significant differences compared with BAV controls $(P<.0125$ after Bonferroni correction).

most prevalent phenotype among patients with BAV with dilated ascending aorta $(82 \%, 22 / 27)$, followed by type 3 $(11 \%, 3 / 27)$ and type $1(7 \%, 2 / 27)$. The distribution of aortopathy phenotypes was similar among all $3 \mathrm{BAV}$ groups.

\section{Systolic Aortic Outflow Patterns}

Representative 4D flow MRI images for systolic 3D blood flow visualization and analysis plane placement are shown in Figure 1. Examples for typical flow patterns observed for the different patient groups are shown in Figure 2. Volunteers with TAV showed a central flow jet and cohesive streamlines directing blood flow parallel to the vessel wall, which indicated high-velocity vectors centering the majority of vessel lumen and decreasing in velocity when approaching the vessel wall. On the other hand, patients with BAV demonstrated eccentric outflow jets accompanied by helical streamlines extending from the aortic root to the origin of aortic arch. These flow patterns contributed to altered flow impingement zones along the ascending aorta and a skewed distribution of systolic velocity vectors (Video 1). For the BAV-AI group, the velocity distribution within ascending aorta was similar to that of the BAV-CTL group but with a universal elevation. For the BAV-AS group, highly eccentric velocity distribution was observed.

\section{Wall Shear Stress Patterns and Flow Eccentricity Assessment}

Circumferentially averaged WSS was significantly elevated in the BAV-CTL group compared with the TAV-CTL group at the level of the proximal $(P=.010)$ and mid-ascending aorta $(P=.009)$. The location of peak systolic WSS migrated along a right-handed helix among all patients with BAV, from the right-anterior of proximal ascending aorta (BAV vs TAV: $0.91 \pm 0.23 \mathrm{~N} / \mathrm{m}^{2}$ vs $\left.0.43 \pm 0.12 \mathrm{~N} / \mathrm{m}^{2}, P<.001\right)$ toward left-anterior at the supra-aortic level (BAV vs TAV: $0.76 \pm 0.20 \mathrm{~N} / \mathrm{m}^{2}$ vs $0.44 \pm 0.07 \mathrm{~N} / \mathrm{m}^{2}, P<.001$ ) (Table 2, Figure 3).

The patterns of WSS distribution along the ascending aorta were distinctly different between BAV-AI and BAV-AS groups. Compared with the BAV-CTL group, the BAV-AI group demonstrated a marked elevation of systolic WSS in all 8 positions for all 3 analysis planes in the ascending aorta (all $P<.01$ ). Circumferentially averaged 

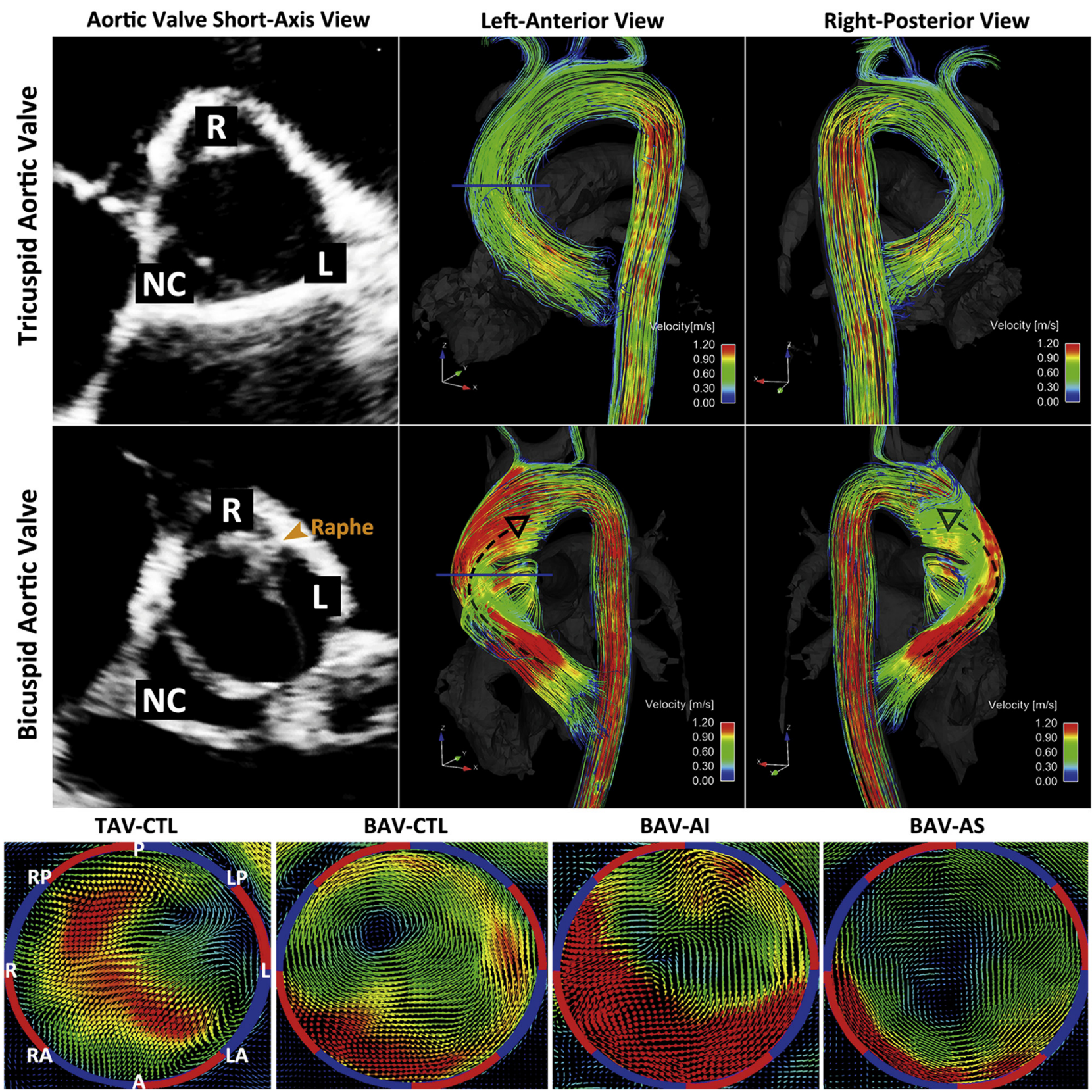

FIGURE 2. Visualization of flow patterns. 3D streamline visualization of peak systolic flow in a patient with BAV of R-L fusion type with no more than mild aortic dysfunction (BAV-CTL, middle) in comparison with an aortic size-matched TAV volunteer (TAV-CTL, top). Note the presence of distinct 3D outflow jet directing toward the right-anterior wall of the ascending aorta via right-handed helix (black dashed arrows) in the R-L BAV case. Bottom shows 3D velocity-coded vectors with uniform velocity limit of $1.2 \mathrm{~m} / \mathrm{s}$ in BAV cases with severe AI, severe AS, BAV-CTL, and TAV-CTL at the mid-ascending aorta. Different distributions of high-velocity vectors can be appreciated between groups in correspondence to differential exertion of shear forces. $R$, Right; $L$, left; $T A V$, tricuspid aortic valve; $B A V$, bicuspid aortic valve; $R P$, right posterior; $P$, posterior; $L P$, left posterior; $L A$, left anterior; $A$, anterior; $R A$, right anterior.

WSS at the level of proximal, middle, and distal ascending aorta was positively correlated with cardiac stroke volume $(r=0.62, P=.014 ; r=0.74, P=.002 ; r=0.68$, $P=.005$; respectively), but not with age, systolic blood pressure, or aortic sinus diameter (all $P>.05$ ). Parameters for flow eccentricity including WSS-SD 8 P and $\mathrm{WSS}_{\text {max-min }}$ ratio were similar to those in the BAV-CTL group (all $P>.10$ ). For the BAV-AS group, regional WSS was elevated only in selected positions, resulting in a highly skewed systolic WSS distribution with elevated WSS-SD $8 \mathrm{P}$ and $\mathrm{WSS}_{\max -\min }$ ratio (both $P<.005$ ). (Table 2, Figure 3). On the basis of the WSS eccentricity threshold defined as RA-LP greater than $0.2 \mathrm{~N} / \mathrm{m}^{2}$ at the level of midascending aorta, eccentric WSS distribution was most 


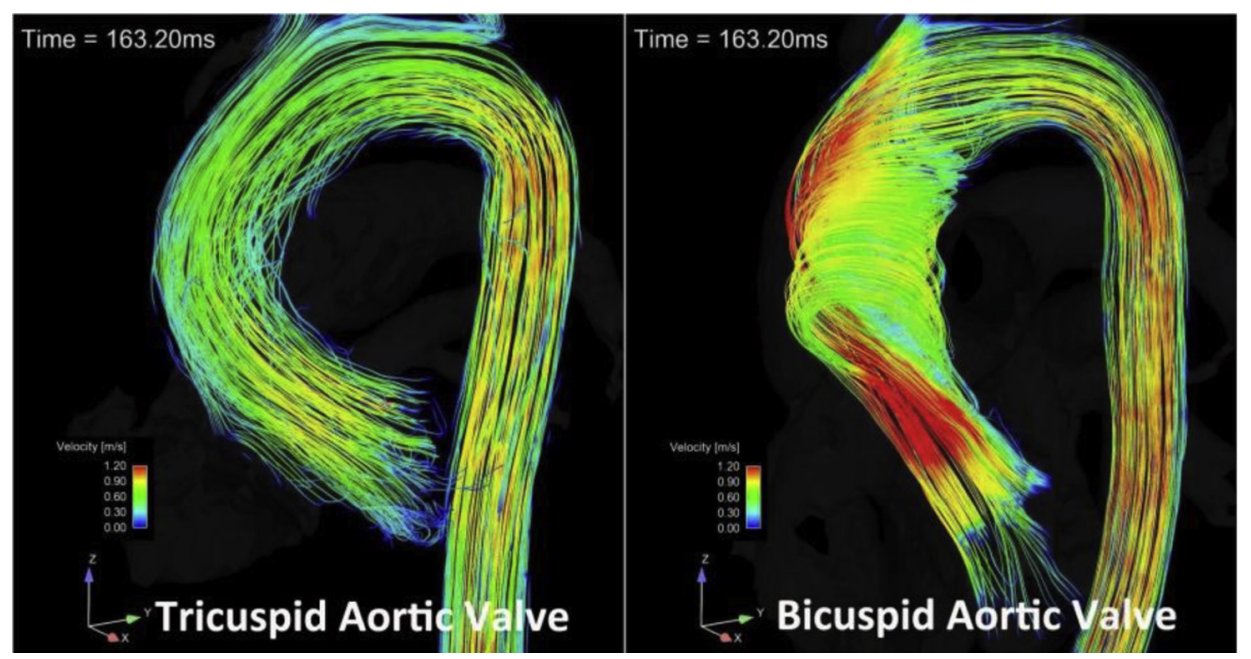

VIDEO 1. Three-dimensional streamline visualization. Highly eccentric outflow jet pattern was found in the BAV case in comparison with cohesive streamlines parallel to the vessel wall in the TAV control case. Video available at: http://www.jtcvsonline.org/article/S0022-5223(17)30191-5/addons.

frequently observed in the BAV-AS group $\left(0.62 \pm 0.22 \mathrm{~N} / \mathrm{m}^{2}, 100 \%\right)$, followed by the BAV-CTL $\left(0.35 \pm 0.17 \mathrm{~N} / \mathrm{m}^{2}, 70.0 \%\right)$ and BAV-AI groups $\left(0.32 \pm 0.19 \mathrm{~N} / \mathrm{m}^{2}, 73.3 \%\right)$. Positive correlations were found between aortic diameter and WSS-SD $8 \mathrm{P}$ at the level of mid-ascending aorta for both the BAV-CTL $(r=0.56$, $P=.010)$ and BAV-AI $(r=0.68, P=.006)$ groups. In addition, there was a positive correlation between WSS-SD ${ }_{8 \mathrm{P}}$ with peak aortic valve velocity $(r=0.92$, $P<.001)$ but not with aortic diameter $(r=0.12, P=.68)$ at the same level in the BAV-AS group (Figure 4). All WSS values and distributions were similar among the 3 BAV groups at the level of proximal descending aorta.

\section{DISCUSSION}

Among the most hazardous complications of BAV, aortopathy attracts both academic and clinical attention for its problematic consequences, intricate management, and relatively unpredictable natural history. From 1997 to 2014, approximately 12 international guidelines have been issued on the threshold for surgical intervention in BAV aortopathy, initiating at a recommended threshold of 5.0 to $5.5 \mathrm{~cm}$ in $1997,{ }^{21,22}$ peaking at a nadir of 4.0 to $5.0 \mathrm{~cm}$ in $2010,{ }^{23}$ and then retreating back to 4.5 to $5.5 \mathrm{~cm}$ in $2014 .{ }^{17}$ Complicating these matters, the shifting criteria are echoed by marked gaps in knowledge and attitudes among cardiovascular surgeons on the management

TABLE 2. Hemodynamic qualification at measurement planes

\begin{tabular}{|c|c|c|c|c|}
\hline & \multicolumn{2}{|c|}{ Control group } & \multicolumn{2}{|c|}{ Study group } \\
\hline & TAV-CTL & BAV-CTL & BAV-AI & BAV-AS \\
\hline Cardiac stroke volume, $\mathrm{mL} / \mathrm{beat}$ & $54.3 \pm 7.5$ & $55.3 \pm 7.9$ & $107.3 \pm 13.8^{*}$ & $59.1 \pm 5.7$ \\
\hline \multicolumn{5}{|l|}{ Systolic WSS, N/m² } \\
\hline Proximal ascending aorta & $0.44 \pm 0.06^{*}$ & $0.53 \pm 0.11$ & $0.69 \pm 0.10^{*}$ & $0.65 \pm 0.11^{*}$ \\
\hline Mid-ascending aorta & $0.44 \pm 0.07 *$ & $0.57 \pm 0.09$ & $0.75 \pm 0.12 *$ & $0.70 \pm 0.11^{*}$ \\
\hline Distal ascending aorta & $0.43 \pm 0.06$ & $0.48 \pm 0.08$ & $0.69 \pm 0.15^{*}$ & $0.61 \pm 0.08^{*}$ \\
\hline Proximal descending aorta & $0.42 \pm 0.05$ & $0.46 \pm 0.07$ & $0.47 \pm 0.05$ & $0.48 \pm 0.10$ \\
\hline WSS eccentricity, n (\%) & $0 *$ & $14(70.0)$ & $11(73.3)$ & $15(100.0)$ \\
\hline \multicolumn{5}{|l|}{ WSS-SD ${ }_{8 \mathrm{P}}, \mathrm{N} / \mathrm{m}^{2}$} \\
\hline Proximal ascending aorta & $0.08 \pm 0.02 *$ & $0.14 \pm 0.05$ & $0.14 \pm 0.04$ & $0.22 \pm 0.07^{*}$ \\
\hline Mid-ascending aorta & $0.08 \pm 0.02 *$ & $0.15 \pm 0.05$ & $0.14 \pm 0.05$ & $0.23 \pm 0.07 *$ \\
\hline Distal ascending aorta & $0.05 \pm 0.01$ & $0.06 \pm 0.02$ & $0.06 \pm 0.02$ & $0.17 \pm 0.05^{*}$ \\
\hline Proximal descending aorta & $0.05 \pm 0.01$ & $0.05 \pm 0.01$ & $0.05 \pm 0.01$ & $0.05 \pm 0.01$ \\
\hline \multicolumn{5}{|l|}{ Ratio $\mathrm{WSS}_{\max -\min }$} \\
\hline Proximal ascending aorta & $1.61(1.53-1.79)^{*}$ & $2.08(1.82-2.41)$ & $1.86(1.44-2.22)$ & $3.00(2.24-4.29)^{*}$ \\
\hline Mid-ascending aorta & $1.65(1.50-2.07)^{*}$ & $2.36(1.75-3.05)$ & $1.76(1.61-2.16)$ & $3.35(2.92-4.08)^{*}$ \\
\hline Distal ascending aorta & $1.43(1.33-1.53)$ & $1.51(1.38-1.73)$ & $1.30(1.23-1.45)$ & $2.62(2.32-3.99)^{*}$ \\
\hline Proximal descending aorta & $1.44(1.34-1.65)$ & $1.40(1.33-1.49)$ & $1.43(1.34-1.50)$ & $1.44(1.38-1.55)$ \\
\hline
\end{tabular}



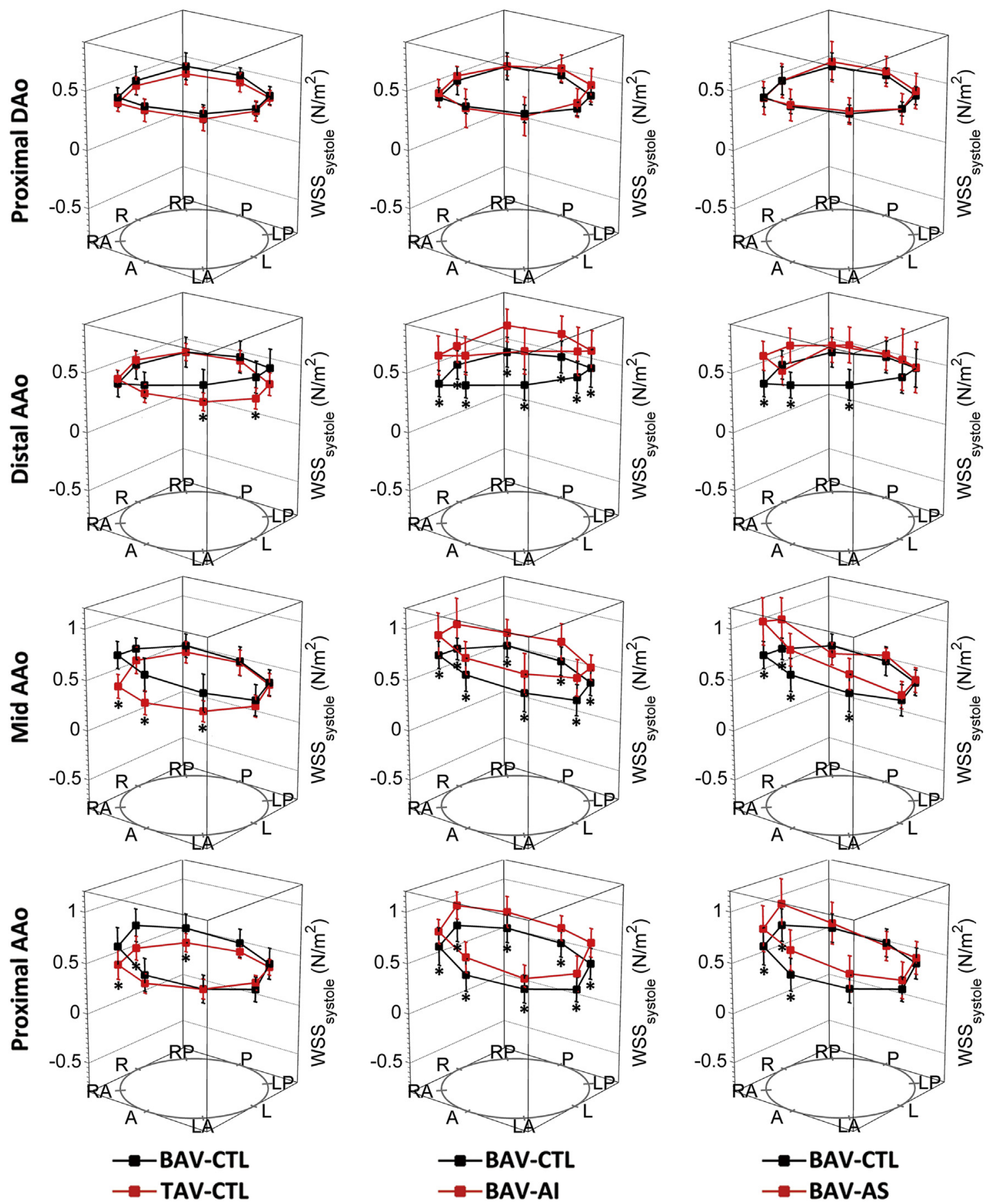

FIGURE 3. Segmental systolic wall shear stress measurements. Segmental systolic WSS measurements were displayed across 8 anatomic positions ( $A$, Anterior; $L$, left; $L A$, left anterior; $L P$, left posterior; $P$, posterior; $R$, right; $R A$, right anterior; $R P$, right posterior) at the proximal ascending aorta, mid-ascending aorta, distal ascending aorta, and proximal descending aorta. Error bars represent standard deviation of individual WSS variation. Asterisks indicate statistical significance $(P<.0125$ after Bonferroni correction) for pairwise comparison among 4 groups. DAo, Descending aorta; $A A o$, ascending aorta.

of BAV aortopathy, including poor adherence to the current guidelines. ${ }^{24}$ Because the majority of patients with BAV are likely to require surgical correction for valve dysfunction in their life, it is vital to reach a uniform consensus on concomitant surgical intervention strategy in patients with BAV with significant aortic insufficiency or stenosis. The present study concentrated exclusively on these patients with BAV and for the first time provided convincing 

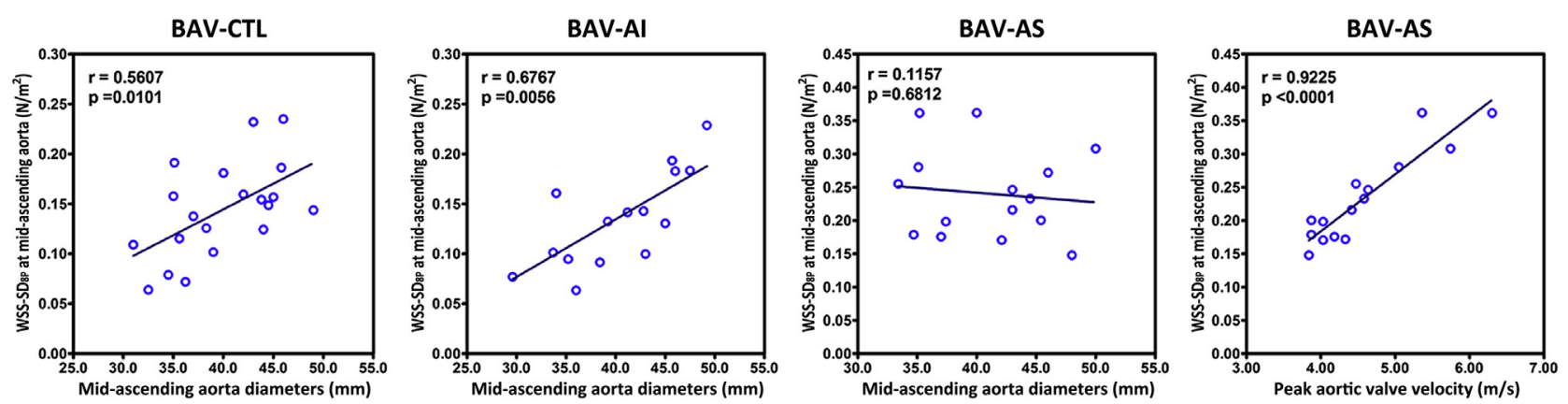

FIGURE 4. Relevant parameters for WSS deviation. Correlation analyses were performed between aortic diameter and $\mathrm{WSS}_{\mathrm{S}} \mathrm{SD}_{8 \mathrm{P}}$ at the level of mid-ascending aorta in BAV-CTL, BAV-AI, and BAV-AS cases. Positive correlation was found between peak aortic valve velocity and WSS-SD 8 p at the mid-ascending aorta in BAV-AS cases. WSS-SD $8 \mathrm{P}$ indicated standard deviation of circumferential wall shear stress across 8 anatomic positions.

evidence to support the hypothesis that aortic valve dysfunction exerts additional but apparently different effects on ascending aorta.

Four-dimensional flow MRI, which permits in vivo assessment of time-resolved 3D blood velocity and enables quantification of complex 3D blood flow pattern in the aorta, provides a comprehensive approach to appreciate the role of hemodynamics in BAV aortopathy. Previous studies have validated the significant differences in systolic aortic flow and WSS patterns between patients with R-L $\mathrm{BAV}$ and patients with right-noncoronary $\mathrm{BAV} .^{10,11}$ Because those with moderate-to-severe aortic insufficiency accounted for less than $10 \%$ in patients with rightnoncoronary BAV, ${ }^{16,25,26}$ we chose patients with R-L BAV as the study population for further categorization based on valve function. We found it similar to previous reports that patients with R-L BAV tended to exhibit peak WSS at the right-anterior or anterior position of ascending aorta, which progressed along a right-handed helical aortic outflow until the level of supra-aortic artery. Of note, the positions of peak WSS along the ascending aorta did not change or exhibit a distinct alteration for the severe aortic insufficiency or stenosis cases. The predominance of type 2 aortopathy also persisted, with or without severe valve dysfunction. On the basis of previous methods that measure systolic WSS as an average over multiple systolic time points to mitigate measurement noise, the magnitude of circumferentially averaged WSS in our study (R-L BAV-CTL: $0.5 \pm 0.1 \mathrm{~N} / \mathrm{m}^{2}$; TAV-CTL: $0.4 \pm 0.1 \mathrm{~N} / \mathrm{m}^{2}$ ) was similar to those previously reported by Mahadevia and colleagues ${ }^{11}$ (R-L BAV-CTL: $0.6 \mathrm{~N} / \mathrm{m}^{2}$; TAV-CTL: 0.3-0.4 N/ $\mathrm{m}^{2}$ ) and Meierhofer and colleagues ${ }^{27}$ (BAV: median $0.6 \mathrm{~N} / \mathrm{m}^{2}$; TAV-CTL: median $0.5 \mathrm{~N} / \mathrm{m}^{2}$ ).

The malformed BAV affects aortic hemodynamics predominantly by altering the direction of outflow jets, which no longer comply with the aortic curvature and result in eccentric WSS distribution along the aortic vessel wall. Aortic valve dysfunction (ie, insufficiency and stenosis) are hypothesized to exert additional but apparently different effects on ascending aorta. For example, regurgitation leads to higher stroke volumes and global aortic outflow velocities, whereas stenosis creates eccentric high-velocity flow jets and regional ascending aortic WSS elevation. ${ }^{2}$ The present study used 4D flow hemodynamics to describe the composite effect of $\mathrm{BAV}$ and $\mathrm{AI} / \mathrm{AS}$ on the ascending aorta and unprecedentedly to advocate this hypothesis in the R-L BAV scenario. To further elaborate, the patients with BAVAI experienced an increased stroke volume, thereby ejecting a larger stroke bolus, which manifested in the form of a universal WSS increase around the ascending aorta instead of exaggerated eccentricity. Aside from age, systolic blood pressure, aortic sinus diameter, and cardiac stroke volume were mostly likely to be accounted for the elevation of circumferentially averaged WSS. Alternatively, for patients with BAV-AS, the diminished aortic valve area and elevated flow velocity further exacerbated the eccentricity of aortic outflow, ${ }^{28,29}$ thus leading to a high WSS-SD 8 P and $\mathrm{WSS}_{\text {max-min }}$ ratio.

The divergence of WSS expression between patients with BAV-AI and patients with BAV-AS reflected the spatiotemporal characteristics of distorted flow in the setting of aortic valve dysfunction, which could have long-term consequences in affected aortic wall tissue. It was argued by Guzzardi and colleagues ${ }^{12}$ that WSS might propel changes in local extracellular matrix homeostasis and ascending aortic wall structure. In aortic regions with elevated WSS, elastin content and structure were severely disrupted compared with distal regions presenting normal WSS. Pathology studies also found worse elastic fiber loss in the aortic wall tissue of patients with BAV-AI, ${ }^{30,31}$ which is in accordance with clinical observations that patients with BAV-AI, especially those with predominantly sinus dilatation (so-called root phenotype), had faster ascending aorta dilatation and higher risk for adverse aortic events even after aortic valve replacement. ${ }^{13-15,32-34}$ In the advent of this evidence, an aggressive policy of preventive aortic intervention might be appreciated in patients with BAV-AI who exhibit a uniform WSS elevation around the lumen of the ascending aorta and who are also known to present at a younger age and with the "root phenotype." 
Meanwhile, patients with BAV-AS who exhibit exaggerated flow eccentricity and elevation of regional WSS could benefit from less-aggressive treatment strategies, including reduction aortoplasty guided by WSS mapping. It was supported by long-term follow-up studies that clinical outcomes of reduction aortoplasty might rely on the extent of localized degeneration of the aortic wall at the greater curvature, ${ }^{35-37}$ which was typically associated with BAV stenosis. Considering the elderly age of patients with BAV-AS, the less-aggressive approach of reduction aortoplasty could be a surgical option for future clinical studies because it was associated with a lower rate of early postoperative complications and shorter intensive care unit and hospital stay. ${ }^{37,38}$

Great efforts have been made to explore ideal quantitative metrics of BAV aortic hemodynamics generated by cardiac magnetic resonance (CMR) to serve as novel risk markers and potential prognostic indicators to better address individual risk stratification in BAV aortopathy. ${ }^{39}$ Hope and colleagues ${ }^{40,41}$ found promising correlation between ascending aortic growth rates and flow eccentricity. Considering the time constraint and technical complexity in WSS quantification, subsequent studies preferred simpler CMR metrics, namely, flow displacement, restrictive leaflet excursion, and angle between the experimental flow vector and the theoretic axis of left ventricular outflow to classic WSS quantification in BAV scenario. ${ }^{11,42-44}$ To better describe shear forces that had well-recognized effects on vascular function and diseases, our study directly used standard deviations of circumferential WSS to reflect flow eccentricity, which increased with ascending aortic diameters in patients with nonstenotic BAV and with peak aortic valve velocity in stenotic cases. Further endeavors such as multicentered collaborations should be conveyed to determine the CMR hemodynamic metrics applicable for cardiovascular surgeons to balance the benefit and risk of preventive aortic intervention among patients with BAV, especially which WSS pattern conferred increased dissection risk and what degree of WSS elevation indicated surgical intervening even if the ascending aorta did not reach $5.5 \mathrm{~cm}$ yet.

\section{Study Limitations}

The present study should be appreciated with knowledge of its limitations, such as a relatively small subject number and exclusion of cases other than R-L cusp fusion type. It should be noted that the results presented in this article have not been adjusted for blood pressure, age, or other patient characteristics, and further studies are warranted to systemically investigate the combined influence of body surface area, blood pressure, and age on the metrics of aortic hemodynamics. Although satisfying data reproducibility was achieved via standardized acquisition and analysis protocols, the technical challenges associated with 4D flow MRI include limited spatial resolution, partial volume effects, and numeric derivation of the velocity field. ${ }^{5}$ The time-consuming acquiring and analyzing process is still a major obstacle for its clinical availability. Computational fluid dynamics is a complementary tool with its own challenges. ${ }^{29}$ Nonetheless, it is a perfect tool to investigate those parameters such as helicity and oscillatory shear index that we cannot easily measure with MRI. Furthermore, in-depth analysis of pathologic and gene-expressing profiles within ascending aortic tissue would help to integrate hemodynamic and genetic knowledge into a complete working tool kit to put better interpretation of the scale in WSS increments on biomechanical changes of actual aortic tissue and possibly to predict BAV aortopathy. Longitudinal studies are needed to understand restoration of hemodynamics and additional aberrations after valve surgery, which is crucial for precise patient management. A detailed paradigm of BAV phenotypes and their association with clinical hazards for aortopathy could benefit interventional strategies and in the process remodel our perception of this highly heterogeneous entity.

\section{CONCLUSIONS}

On the basis of skewed WSS distribution in patients with R-L BAV, 4D flow MRI revealed marked hemodynamic alterations in the ascending aorta of those with BAV-AI and BAV-AS. In proportion to increased stroke volume, a universal elevation of WSS was found at the vessel wall in patients with BAV-AI. On the contrary, an increased WSS burden was found in BAV-AS cases in the form of a focal elevation of flow eccentricity due to diminished valve area and accelerated flow velocity. Hemodynamic markers derived from CMR metrics represent promising developments toward establishing noninvasive indicators for risk stratification and management of patients with BAV.

\section{Conflict of Interest Statement}

Authors have nothing to disclose with regard to commercial support.

The authors thank Dr Caixia Fu from Siemens Shenzhen Magnetic Resonance for assistance in MRI data acquiring.

\section{References}

1. Fedak PW, Verma S, David TE, Leask RL, Weisel RD, Butany J. Clinical and pathophysiological implications of a bicuspid aortic valve. Circulation. 2002; 106:900-4.

2. Tadros TM, Klein MD, Shapira OM. Ascending aortic dilatation associated with bicuspid aortic valve: pathophysiology, molecular biology, and clinical implications. Circulation. 2009;119:880-90.

3. Wang Y, Wu B, Dong L, Wang C, Shu X. Type A aortic dissection in patients with bicuspid or tricuspid aortic valves: a retrospective comparative study in 288 Chinese patients. Eur J Cardiothorac Surg. 2013;44:172-7.

4. Verma S, Siu SC. Aortic dilatation in patients with bicuspid aortic valve. $N$ Engl J Med. 2014;370:1920-9

5. Spinale FG, Bolger AF. Fate versus flow: wall shear stress in the aortopathy associated with bicuspid aortic valves. J Am Coll Cardiol. 2015;66:901-4. 
6. Opotowsky AR, Perlstein T, Landzberg MJ, Colan SD, O'Gara PT, Body SC, et al. A shifting approach to management of the thoracic aorta in bicuspid aortic valve. J Thorac Cardiovasc Surg. 2013;146:339-46.

7. Hardikar AA, Marwick TH. The natural history of guidelines: the case of aortopathy related to bicuspid aortic valves. Int J Cardiol. 2015;199:150-3.

8. Prakash SK, Bossé Y, Muehlschlegel JD, Michelena HI, Limongelli G, Della Corte A, et al. A roadmap to investigate the genetic basis of bicuspid aortic valve and its complications: insights from the International BAVCon (Bicuspid Aortic Valve Consortium). J Am Coll Cardiol. 2014;64:832-9.

9. Hope MD, Hope TA, Meadows AK, Ordovas KG, Urbania TH, Alley MT, et al. Bicuspid aortic valve: four-dimensional MR evaluation of ascending aortic systolic flow patterns. Radiology. 2010;255:53-61.

10. Barker AJ, Markl M, Bürk J, Lorenz R, Bock J, Bauer S, et al. Bicuspid aortic valve is associated with altered wall shear stress in the ascending aorta. Circ Cardiovasc Imaging. 2012;5:457-66.

11. Mahadevia R, Barker AJ, Schnell S, Entezari P, Kansal P, Fedak PW, et al. Bicuspid aortic cusp fusion morphology alters aortic three-dimensional outflow patterns, wall shear stress, and expression of aortopathy. Circulation. 2014;129: 673-82.

12. Guzzardi DG, Barker AJ, van Ooij P, Malaisrie SC, Puthumana JJ, Belke DD, et al. Valve-related hemodynamics mediate human bicuspid aortopathy: insights from wall shear stress mapping. J Am Coll Cardiol. 2015;66:892-900.

13. Stephens EH, Hope TA, Kari FA, Kvitting JP, Liang DH, Herfkens RJ, et al. Greater asymmetric wall shear stress in Sievers' type 1/LR compared with 0/LAT bicuspid aortic valves after valve-sparing aortic root replacement. J Thorac Cardiovasc Surg. 2015;150:59-68.

14. Girdauskas E, Disha K, Raisin HH, Secknus MA, Borger MA, Kuntze T. Risk of late aortic events after an isolated aortic valve replacement for bicuspid aortic valve stenosis with concomitant ascending aortic dilation. Eur J Cardiothorac Surg. 2012;42:832-8.

15. Sievers HH, Stierle U, Mohamed SA, Hanke T, Richardt D, Schmidtke C, et al. Toward individualized management of the ascending aorta in bicuspid aortic valve surgery: the role of valve phenotype in 1362 patients. J Thorac Cardiovasc Surg. 2014;148:2072-80.

16. Wang Y, Wu B, Li J, Dong L, Wang C, Shu X. Impact of aortic insufficiency on ascending aortic dilatation and adverse aortic events after isolated aortic valve replacement in patients with a bicuspid aortic valve. Ann Thorac Surg. 2016; 101:1707-14.

17. Nishimura RA, Otto CM, Bonow RO, Carabello BA, Erwin JP III, Guyton RA, et al. 2014 AHA/ACC guideline for the management of patients with valvular heart disease: a report of the American College of Cardiology/American Heart Association Task Force on Practice Guidelines. J Thorac Cardiovasc Surg. 2014;148:e1-132.

18. Lang RM, Badano LP, Mor-Avi V, Afilalo J, Armstrong A, Ernande L, et al. Recommendations for cardiac chamber quantification by echocardiography in adults: an update from the American Society of Echocardiography and the European Association of Cardiovascular Imaging. J Am Soc Echocardiogr. 2015;28:1-39.

19. Sievers HH, Schmidtke C. A classification system for the bicuspid aortic valve from 304 surgical specimens. J Thorac Cardiovasc Surg. 2007;133:1226-33.

20. Erbel R, Alfonso F, Boileau C, Dirsch O, Eber B, Haverich A, et al. Diagnosis and management of aortic dissection. Eur Heart J. 2001;22:1642-81.

21. Kouchoukos NT, Dougenis D. Surgery of the thoracic aorta. N Engl J Med. 1997; 336:1876-88.

22. Coady MA, Rizzo JA, Hammond GL, Mandapati D, Darr U, Kopf GS, et al. What is the appropriate size criterion for resection of thoracic aortic aneurysms? Thorac Cardiovasc Surg. 1997;113:476-91.

23. Hiratzka LF, Bakris GL, Beckman JA, Bersin RM, Carr VF, Casey DE Jr, et al. 2010 ACCF/AHA/AATS/ACR/ASA/SCA/SCAI/SIR/STS/SVM guidelines for the diagnosis and management of patients with Thoracic Aortic Disease: a report of the American College of Cardiology Foundation/American Heart Association Task Force on Practice Guidelines, American Association for Thoracic Surgery, American College of Radiology, American Stroke Association, Society of Cardiovascular Anesthesiologists, Society for Cardiovascular Angiography and Interventions, Society of Interventional Radiology, Society of Thoracic Surgeons, and Society for Vascular Medicine. Circulation. 2010;121:e266-369.

24. Verma S, Yanagawa B, Kalra S, Ruel M, Peterson MD, Yamashita MH, et al. Knowledge, attitudes, and practice patterns in surgical management of bicuspid aortopathy: a survey of 100 cardiac surgeons. J Thorac Cardiovasc Surg. 2013; 146:1033-40.

25. Kang JW, Song HG, Yang DH, Baek S, Kim DH, Song JM, et al. Association between bicuspid aortic valve phenotype and patterns of valvular dysfunction and bicuspid aortopathy: comprehensive evaluation using MDCT and echocardiography. JACC Cardiovasc Imaging. 2013;6:150-61.

26. Sievers HH, Stierle U, Hachmann RM, Charitos EI. New insights in the association between bicuspid aortic valve phenotype, aortic configuration and valve haemodynamics. Eur J Cardiothorac Surg. 2016;49:439-46.

27. Meierhofer C, Schneider EP, Lyko C, Hutter A, Martinoff S, Markl M, et al. Wall shear stress and flow patterns in the ascending aorta in patients with bicuspid aortic valves differ significantly from tricuspid aortic valves: a prospective study. Eur Heart J Cardiovasc Imaging. 2013;14:797-804.

28. Bissel MM, Hess AT, Biasiolli L, Glaze SJ, Loudon M, Pitcher A, et al. Aortic dilation in bicuspid aortic valve disease: flow pattern is a major contributor and differs with valve fusion type. Circ Cardiovasc Imaging. 2013;6:499-507.

29. Youssefi P, Gomez A, He T, Anderson L, Bunce N, Sharma R, et al. Patient-specific computational fluid dynamics — assessment of aortic hemodynamics in a spectrum of aortic valve pathologies. J Thorac Cardiovasc Surg. 2017;153:8-20.e3.

30. Roberts WC, Vowels TJ, Ko JM, Filardo G, Hebeler RF Jr, Henry AC, et al. Comparison of the structure of the aortic valve and ascending aorta in adults having aortic valve replacement for aortic stenosis versus for pure aortic regurgitation and resection of the ascending aorta for aneurysm. Circulation. 2011;123:896-903.

31. Girdauskas E, Rouman M, Borger MA, Kuntze T. Comparison of aortic media changes in patients with bicuspid aortic valve stenosis versus bicuspid valve insufficiency and proximal aortic aneurysm. Interact Cardiovasc Thorac Surg. 2013;17:931-6.

32. Roberts WC. Prophylactic replacement of a dilated ascending aorta at the time of aortic valve replacement of a dysfunctioning congenitally unicuspid or bicuspid aortic valve. Am J Cardiol. 2011;108:1371-2.

33. Della Corte A, Bancone C, Buonocore M, Dialetto G, Covino FE, Manduca S, et al. Pattern of ascending aortic dimensions predicts the growth rate of the aorta in patients with bicuspid aortic valve. JACC Cardiovasc Imaging. 2013;6: 1301-10.

34. Detaint D, Michelena HI, Nkomo VT, Vahanian A, Jondeau G, Sarano ME. Aortic dilatation patterns and rates in adults with bicuspid aortic valves: a comparative study with Marfan syndrome and degenerative aortopathy. Heart. 2014;100:126-34

35. Tsamis A, Phillippi JA, Koch RG, Chan PG, Krawiec JT, D'Amore A, et al. Extracellular matrix fiber microarchitecture is region-specific in bicuspid aortic valve-associated ascending aortopathy. J Thorac Cardiovasc Surg. 2016;151: 1718-28.e5.

36. Fedak PW, Guzzardi DG. Real estate of the bicuspid aorta: location, location, location! J Thorac Cardiovasc Surg. 2016;151:1728-9.

37. Della Corte A, De Feo M, Bancone C, Provenzano R, Giordano S, Buonocore M, et al. Long-term follow-up of reduction ascending aortoplasty with autologous partial wrapping: for which patient is waistcoat aortoplasty best suited? Interact Cardiovasc Thorac Surg. 2012; 14:56-63.

38. Della Corte A, Body SC, Booher AM, Schaefers HJ, Milewski RK, Michelena HI, et al. Surgical treatment of bicuspid aortic valve disease: knowledge gaps and research perspectives. J Thorac Cardiovasc Surg. 2014;147: $1749-57$.

39. Fedak PW, Barker AJ, Verma S. Year in review: bicuspid aortopathy. Curr Opin Cardiol. 2016;31:132-8.

40. Hope MD, Hope TA, Crook SE, Ordovas KG, Urbania TH, Alley MT, et al. 4D flow CMR in assessment of valve-related ascending aortic disease. JACC Cardiovasc Imaging. 2011;4:781-7.

41. Hope MD, Wrenn J, Sigovan M, Foster E, Tseng EE, Saloner D. Imaging biomarkers of aortic disease: increased growth rates with eccentric systolic flow. J Am Coll Cardiol. 2012;60:356-7.

42. Della Corte A, Bancone C, Conti CA, Votta E, Redaelli A, Del Viscovo L, et al. Restricted cusp motion in right-left type of bicuspid aortic valves: a new risk marker for aortopathy. J Thorac Cardiovasc Surg. 2012;144:360-9.

43. den Reijer PM, Sallee D III, van der Velden P, Zaaijer ER, Parks WJ, Ramamurthy S, et al. Hemodynamic predictors of aortic dilatation in bicuspid aortic valve by velocity-encoded cardiovascular magnetic resonance. $J$ Cardiovasc Magn Reson. 2010;12:4.

44. Garcia J, Barker AJ, Murphy I, Jarvis K, Schnell S, Collins JD, et al. Four-dimensional flow magnetic resonance imaging-based characterization of aortic morphometry and haemodynamics: impact of age, aortic diameter, and valve morphology. Eur Heart J Cardiovasc Imaging. 2016;17:877-84.

Key Words: bicuspid aortic valve, magnetic resonance imaging, wall shear stress 


\section{APPENDIX E1. FORMULATION OF WALL SHEAR STRESS}

Wall shear stress (WSS) depends on the spatial velocity gradient at the vessel wall; therefore, can be calculated from the 3-directional velocity fields measured by 4D flow MRI. ${ }^{\mathrm{E} 1}$ It should be noted that the term "WSS" is used to describe WSS estimations based on the interpolation of local velocity derivatives onto the manually drawn vessel lumen contours as described by Stalder et al. ${ }^{\mathrm{E} 2}$ Briefly, B-spline interpolation allows for the calculation of the local blood flow velocity derivatives on the vessel contour and thus allows a direct estimate of time-resolved segmental WSS vectors to be obtained independently of any global assumptions regarding the flow profile.

The WSS vector $(\vec{\tau})$ for a Newtonian incompressible fluid can be derived from the velocity field based on the deformation tensor at the vessel wall:

$$
\begin{aligned}
& \vec{\tau}=2 \eta \dot{\varepsilon} \cdot \vec{n} \\
& =\eta \cdot\left(\begin{array}{c}
2 n_{1} \frac{\partial v_{1}}{\partial x_{1}}+n_{2}\left(\frac{\partial \mathrm{v}_{1}}{\partial \mathrm{x}_{2}}+\frac{\partial \mathrm{v}_{2}}{\partial \mathrm{x}_{1}}\right)+n_{3}\left(\frac{\partial \mathrm{v}_{1}}{\partial \mathrm{x}_{3}}+\frac{\partial \mathrm{v}_{3}}{\partial \mathrm{x}_{1}}\right) \\
n_{1}\left(\frac{\partial \mathrm{v}_{1}}{\partial \mathrm{x}_{2}}+\frac{\partial \mathrm{v}_{2}}{\partial \mathrm{x}_{1}}\right)+2 n_{2} \frac{\partial v_{2}}{\partial x_{2}}+n_{3}\left(\frac{\partial \mathrm{v}_{2}}{\partial \mathrm{x}_{3}}+\frac{\partial \mathrm{v}_{3}}{\partial \mathrm{x}_{2}}\right) \\
n_{1}\left(\frac{\partial \mathrm{v}_{1}}{\partial \mathrm{x}_{3}}+\frac{\partial \mathrm{v}_{3}}{\partial \mathrm{x}_{1}}\right)+n_{2}\left(\frac{\partial \mathrm{v}_{2}}{\partial \mathrm{x}_{3}}+\frac{\partial \mathrm{v}_{3}}{\partial \mathrm{x}_{2}}\right)+2 n_{3} \frac{\partial v_{3}}{\partial x_{3}}
\end{array}\right)
\end{aligned}
$$

$\eta$ : viscosity, $\dot{\varepsilon}$ : deformation tensor, $\vec{n}$ : inward unit normal and the deformation tensor, $\mathrm{v}$ : velocity components, $\mathrm{x}$ : the spatial dimensions; [1,2,3]: 3 orthogonal coordinates.

To calculate $\vec{\tau}$ from $2 \mathrm{D}$ data with three-directional velocity encoding, it was assumed that the 2D analysis plane was normal to the vessel surface, that is, $\vec{n}=\left(n_{1}, n_{2}, 0\right)$. No flow through the vessel condition was enforced. This implies $\vec{n} \cdot \frac{\partial \vec{v}}{\partial \mathrm{x}_{3}}=n_{1} \frac{\partial \mathrm{v}_{1}}{\partial \mathrm{x}_{3}}+n_{2} \frac{\partial \mathrm{v}_{2}}{\partial \mathrm{x}_{3}}=0$, and finally:

$$
\vec{\tau}=\eta \cdot\left(\begin{array}{c}
2 n_{1} \frac{\partial v_{1}}{\partial x_{1}}+n_{2}\left(\frac{\partial \mathrm{v}_{1}}{\partial \mathrm{x}_{2}}+\frac{\partial \mathrm{v}_{2}}{\partial \mathrm{x}_{1}}\right) \\
n_{1}\left(\frac{\partial \mathrm{v}_{1}}{\partial \mathrm{x}_{2}}+\frac{\partial \mathrm{v}_{2}}{\partial \mathrm{x}_{1}}\right)+2 n_{2} \frac{\partial v_{2}}{\partial x_{2}} \\
n_{1} \frac{\partial v_{3}}{\partial x_{1}}+n_{2} \frac{\partial v_{3}}{\partial x_{2}}
\end{array}\right)
$$

which can be used to calculate vectorial WSS from data of transversal planes with three-directional velocity information.

\section{E-References}

E1. Markl M, Chan FP, Alley MT, Wedding KL, Draney MT, Elkins CJ, et al Time-resolved three-dimensional phase-contrast MRI. J Magn Reson Imaging. 2003;17:499-506.

E2. Stalder AF, Russe MF, Frydrychowicz A, Bock J, Hennig J, Markl M Quantitative 2D and 3D phase contrast MRI: optimized analysis of blood flow and vessel wall parameters. Magn Reson Med. 2008;60:1218-31. 